\title{
The Wiener Index of an Undirected Power Graph
}

\author{
Volkan Aşkin, Şerife Büyükköse* \\ Gazi University, Mathematics, Ankara, Turkey \\ Email: volkan.askin@gazi.edu.tr, *sbuyukkose@gazi.edu.tr
}

How to cite this paper: Asskin, V. and Büyükköse, S. (2021) The Wiener Index of an Undirected Power Graph. Advances in Linear Algebra \& Matrix Theory, 11, 21-29. https://doi.org/10.4236/alamt.2021.111003

Received: January 25, 2021

Accepted: March 9, 2021

Published: March 12, 2021

Copyright $\odot 2021$ by author(s) and Scientific Research Publishing Inc. This work is licensed under the Creative Commons Attribution International License (CC BY 4.0).

http://creativecommons.org/licenses/by/4.0/

\section{Abstract}

The undirected power graph $P\left(Z_{n}\right)$ of a finite group $Z_{n}$ is the graph with vertex set $G$ and two distinct vertices $u$ and $v$ are adjacent if and only if $u \neq v$ and $\langle u\rangle \subseteq\langle v\rangle$ or $\langle v\rangle \subseteq\langle v\rangle$. The Wiener index $W\left(P\left(Z_{n}\right)\right)$ of an undirected power graph $P\left(Z_{n}\right)$ is defined to be sum $\frac{1}{2} \sum_{\{u, v\} \subseteq V\left(P\left(Z_{n}\right)\right)} d(u, v)$ of distances between all unordered pair of vertices in $P\left(Z_{n}\right)$. Similarly, the edge-Wiener index $W_{e}\left(P\left(Z_{n}\right)\right)$ of $P\left(Z_{n}\right)$ is defined to be the sum $\frac{1}{2} \sum_{e, f} d(e, f)$ of distances between all unordered pairs of edges in $P\left(Z_{n}\right)$. In this paper, we concentrate on the wiener index of a power graph $P\left(Z_{P^{k}}\right)$, $P\left(Z_{p q}\right)$ and $P\left(Z_{p}\right)$. Firstly, we obtain new results on the wiener index and edge-wiener index of power graph $P\left(Z_{n}\right)$, using $m, n$ and Euler $\varphi$ function. Also, we obtain an equivalence between the edge-wiener index and wiener index of a power graph of $Z_{n}$.

\section{Keywords}

Wiener Index, Edge-Wiener Index, An Undirected Power Graph, Line Graph

\section{Introduction}

We define an undirected power graph $P(G)$ for a group $G$ as follows. Let us denote the cylic subgroup genarated by $u \in G$ by $\langle u\rangle$, that is, $\langle u\rangle=\left\{u^{m} \mid m \in \mathbb{N}\right\}$, where $\mathbb{N}$ denotes the set of naturel numbers. The graph $P(G)$ is an undirected graph where vertex set is $G$ and two vertices $u, v \in G$ are adjacent if and only if $u \neq v$ and $\langle u\rangle \subseteq\langle v\rangle$ or $\langle v\rangle \subseteq\langle v\rangle$ (which is equivalent to say $u \neq v$ and $u^{m}=v$ or $v^{m}=u$ for some positive integer m.) [1] [2] [3] [4]. 
For a graph $G$, let $\operatorname{deg}(u)$ and $d(u, v)$ denote the degree of a vertex $u \in V(G)$ and the distance between vertices $u, v \in V(G)$, respectively. Let $L(G)$ denote the line graph of $G$, that is, the graph with vertex set $E(G)$ and two distinct edges $e, f \in E(G)$ adjacent in $L(G)$ whenever they share an end-vertex in $G$. Furthermore, for, $f \in E(G)$, we let $d(e, f)$ denote the distance between $e$ and $f$ in the line graph $L(G)$.

We consider the power graph $P\left(Z_{n}\right)$ for the additive group $Z_{n}$ of integers modulo $n$. The diameter of a graph $G$ is the greatest distance between any pair of vertices, and denoted by $\operatorname{diam}(G)$. In $P\left(Z_{n}\right)$, the distance is one if the vertices is adjacent and the distance is two if the vertices is non adjacent. Therefore, $\operatorname{diam}\left(P\left(Z_{n}\right)\right)=2$. The order an element $\bar{g}$ in $Z_{n}$ is denoted by $(\bar{g})$ or $|g|$. For a positive integer $n, \phi(n)$ denotes the Euler's totient function of $n$.

In this paper, the wiener index and the edge-wiener index, denoted by $W(G)$ and $W_{e}(G)$, respectively and they are defined as follows:

$$
\begin{aligned}
W(G) & =\frac{1}{2} \sum_{\{u, v\} \subseteq V(G)} d(u, v) \\
W_{e}(G) & =\frac{1}{2} \sum_{\{e, f\} \subseteq E(G)} d(e, f)
\end{aligned}
$$

Now, we give some theorem and corollary in literature. Using our main theorems;

Theorem 1. ([5]) For each finite group, the number of edges of the undirected power graph $P(G)$ is given by the formula

$$
E(P(G))=\frac{1}{2} \sum_{g \in G}\{2 o(g)-\phi(o(g))-1\}
$$

Corollary 2. ([6]) The number of edges of the undirected power graph $P\left(Z_{n}\right)$ is given by $\frac{1}{2} \sum_{d \mid n}\{2 d-\phi(d)-1\} \phi(d)$.

Theorem 3. ([3]) Let $G$ be connected graph with $n$ vertices and $m$ edges. If $\operatorname{diam}(G) \leq 2$, Then $W(G)=n(n-1)-m$.

Theorem 4. ([5]) A finite group has a complete undirected power graph if and only if it is cyclic and has order equal to $p^{k}$, where $p$ is a prime and $k$ is a nonnegative integer.

\section{Main Results}

In this section, our aim is to give our main results on the Wiener index and the edge-Wiener index of an undirected power graph $P\left(Z_{n}\right)$ for $n=p^{k}$, or $n=p q$, where $p$ and $q$ are distinct prime numbers and $k$ is a nonnegative integer.

Theorem 5. Let $P\left(Z_{n}\right)$ be an undirected power graph of with $n$ vertices and medges. Then

$$
W\left(P\left(Z_{n}\right)\right)=\frac{1}{2} \sum_{\{u, v\} \subseteq V\left(P\left(Z_{n}\right)\right)} \begin{cases}1, & u \sim v \\ 2, & u \nsim v\end{cases}
$$

Proof. Let 
$R=\left\{\{u, v\} \subseteq V\left(P\left(Z_{n}\right)\right) \mid u \sim v\right.$ if only if $u \neq v,\langle u\rangle \subseteq\langle v\rangle$ or $\left.\langle v\rangle \subseteq\langle u\rangle\right\}$ be a set. In $P\left(Z_{n}\right)$, for $\{u, v\} \subseteq V\left(P\left(Z_{n}\right)\right)$, there are two cases; If $u \nsim v$ then $d(u, v)=2$. Otherwise, i.e. $u \sim v$, then $d(u, v)=1$. Therefore

$$
\begin{aligned}
W\left(P\left(Z_{n}\right)\right) & =\frac{1}{2} \sum_{\{u, v\} \subseteq V\left(P\left(Z_{n}\right)\right)} d(u, v) \\
& =\frac{1}{2}\left(\sum_{\{u, v\} \subseteq R} d(u, v)+\sum_{\{u, v\} \varsubsetneqq R} d(u, v)\right) \\
& =\frac{1}{2} \sum_{\{u, v\} \subseteq R} 1+\frac{1}{2} \sum_{\{u, v\} \subseteq R} 2 \\
& =\frac{1}{2} \sum_{\{u, v\} \subseteq V\left(P\left(Z_{n}\right)\right)}\left\{\begin{array}{l}
1,\{u, v\} \subseteq R \\
2,\{u, v\} \varsubsetneqq R
\end{array}\right.
\end{aligned}
$$

For definition of $R$, we obtain. Thus

$$
W(G)=\frac{1}{2} \sum_{\{u, v\} \subseteq V\left(P\left(Z_{n}\right)\right)} \begin{cases}1, & u \sim v \\ 2, & u \nsim v\end{cases}
$$

the proof is complete.

Corollary 6. Let $p$ and $k$ is prime number and nonnegative integer, respectively. For $P\left(Z_{p^{k}}\right)$ power graph of order $p^{k}$ and $m$ edges,

$$
W\left(P\left(Z_{p^{k}}\right)\right)=\left(\begin{array}{c}
p^{k} \\
2
\end{array}\right) .
$$

Proof. In [2], If $n=p^{k}$ then $P\left(Z_{n}\right)=K_{n}$. For any $u \in V\left(Z_{p^{k}}\right)$, $d(u)=p^{k}-1$.

$$
R^{c}=\left\{\{u, v\} \subseteq V\left(P\left(Z_{n}\right)\right) \mid u \nsim v\right\}=\varnothing
$$

Thus

$$
\begin{aligned}
W\left(P\left(Z_{p^{k}}\right)\right) & =\frac{1}{2} \sum_{\{u, v\} \subseteq V\left(P\left(Z_{n}\right)\right)} \begin{cases}1, & u \sim v \\
2, & u \nsim v\end{cases} \\
& =\frac{1}{2}\left(\sum_{\{u, v\} \subseteq R} d(u, v)+\sum_{\{u, v\} \subseteq \varnothing} d(u, v)\right) \\
& =\frac{1}{2}\left(\sum_{\{u, v\} \subseteq R} 1+\sum_{\{u, v\} \subseteq \varnothing} d(u, v)\right) \\
& =\frac{1}{2} \sum_{\{u, v\} \subseteq R} 1=\frac{1}{2} p^{k}\left(p^{k}-1\right)=\left(\begin{array}{c}
p^{k} \\
2
\end{array}\right)
\end{aligned}
$$

Therefore the proof is proved.

Theorem 7. Let $P\left(Z_{n}\right)$ be a power graph of with $n$ vertices and $m$ edges. Then

$$
W\left(P\left(Z_{n}\right)\right)=\frac{1}{2}\left\{\left(\begin{array}{c}
2 n \\
2
\end{array}\right)+\sum_{g=0}^{n-1}(\phi(|\bar{g}|)-2|\bar{g}|)\right\}
$$

Proof. If we consider Theorem 3. for $=P\left(Z_{n}\right)$, we write

$$
W\left(P\left(Z_{n}\right)\right)=n(n-1)-m
$$




$$
m=\frac{1}{2} \sum_{g \in Z_{n}}\{2 o(g)-\phi(o(g))-1\} .
$$

If we put the value of $m$ into the formula, we obtain

$$
\begin{aligned}
& W\left(P\left(Z_{n}\right)\right)=n(n-1)-m \\
&=n(n-1)-\frac{1}{2} \sum_{g \in Z_{n}}\{2 o(g)-\phi(o(g))-1\} \\
&=n^{2}-n+\frac{1}{2} \sum_{g \in Z_{n}}\{\phi(o(g))-2 o(g)\}-\frac{1}{2} \sum_{g \in Z_{n}} 1 \\
&=n^{2}-n+\frac{n}{2}+\frac{1}{2} \sum_{g \in Z_{n}}\{\phi(o(g))-2 o(g)\} \\
&=\left\{n^{2}-\frac{n}{2}+\frac{1}{2} \sum_{g=0}^{n-1}(\phi(|\bar{g}|)-2|\bar{g}|)\right\} \\
& W\left(P\left(Z_{n}\right)\right)=\frac{1}{2}\left\{\left(\begin{array}{c}
2 n \\
2
\end{array}\right)+\sum_{g=0}^{n-1}(\phi(|\bar{g}|)-2|\bar{g}|)\right\}
\end{aligned}
$$

Thus, the proof is complete.

Corollary 8. Let $P\left(Z_{n}\right)$ be a power graph of with $n=p$, where $p$ is a prime number. Then

$$
W\left(P\left(Z_{n}\right)\right)=\left(\begin{array}{l}
P \\
2
\end{array}\right) .
$$

Proof. Let $n=p$ be a prime number. Then

$$
\begin{aligned}
& W\left(P\left(Z_{p}\right)\right)=\frac{1}{2}\left\{\left(\begin{array}{c}
2 p \\
2
\end{array}\right)+\sum_{g=0}^{p-1}(\phi(|\bar{g}|)-2|\bar{g}|)\right\} \\
& =\frac{1}{2}\left[\frac{2 p(2 p-1)}{2}+\phi(|\overline{0}|)+\phi(|\overline{1}|)+\cdots+\phi(|\overline{p-1}|)-2(|\overline{0}|+|\overline{1}|+\cdots+|\overline{p-1}|)\right] \\
& =\frac{1}{2}\left[2 p^{2}-p-1+(\phi(|\overline{1}|)+\cdots+\phi(|\overline{p-1}|))-2(|\overline{1}|+\cdots+|\overline{p-1}|)\right] \\
& =\frac{1}{2}\left[2 p^{2}-p-1+(p-1) \phi(p)-2(p-1) p\right] \\
& =\frac{1}{2}\left[2 p^{2}-p-1+(p-1)^{2}-2 p^{2}+2 p\right]=\left(\begin{array}{c}
p \\
2
\end{array}\right)
\end{aligned}
$$

Theorem 9. Let $P\left(Z_{n}\right)$ be a power graph of with $n$ vertices and m edges. Then

$$
W\left(P\left(Z_{n}\right)\right)=\frac{1}{2}\left\{\left(\begin{array}{c}
2 n \\
2
\end{array}\right)+\sum_{d \mid n} \phi(d)(\phi(d)-2 d)\right\} .
$$

Proof. Where $P\left(Z_{n}\right)$ is power graph $=P\left(Z_{n}\right)$, using theorem 3. And corollary 2 , we obtain

$$
\begin{gathered}
W\left(P\left(Z_{n}\right)\right)=n(n-1)-m \\
m=\frac{1}{2} \sum_{d \mid n}\{2 d-\phi(d)-1\} \phi(d)
\end{gathered}
$$


If we write this $m$ in formula for $W\left(P\left(Z_{n}\right)\right)$

$$
\begin{aligned}
W\left(P\left(Z_{n}\right)\right) & =n(n-1)-m \\
& =n(n-1)-\frac{1}{2} \sum_{d \mid n}\{2 d-\phi(d)-1\} \phi(d) \\
& =n^{2}-n+\frac{1}{2} \sum_{d \mid n} \phi(d)^{2}+\frac{1}{2} \sum_{d \mid n} \phi(d)-\sum_{d \mid n} d \phi(d) \\
& =n^{2}-\frac{n}{2}+\frac{1}{2} \sum_{d \mid n} \phi(d)(\phi(d)-2 d) \\
W\left(P\left(Z_{n}\right)\right) & =\frac{1}{2}\left\{\left(\begin{array}{c}
2 n \\
2
\end{array}\right)+\sum_{d \mid n} \phi(d)(\phi(d)-2 d)\right\} .
\end{aligned}
$$

End of proof.

Corollary 10. Let $P\left(Z_{n}\right)$ be a power graph of with $n=p q$ vertices and $m$ edges, where $p$ and $q$ are distinct prime numbers. Then

$$
W\left(P\left(Z_{p q}\right)\right)=m+2 \phi(p q)
$$

or equiently

$$
W\left(P\left(Z_{p q}\right)\right)=\left(\begin{array}{c}
p q \\
2
\end{array}\right)+\phi(p q)
$$

Proof. If we write $n=p q$ in theorem 9., we obtain

$$
\begin{aligned}
W\left(P\left(Z_{p q}\right)\right)= & \frac{1}{2}\left\{\left(\begin{array}{c}
2 p q \\
2
\end{array}\right)+\sum_{d \mid p q} \phi(d)(\phi(d)-2 d)\right\} \\
= & \frac{1}{2}[p q(2 \cdot p q-1)+\phi(1)(\phi(1)-2 \cdot 1)+\phi(p)(\phi(p)-2 \cdot p) \\
& +\phi(q)(\phi(q)-2 \cdot q)+\phi(p q)(\phi(p q)-2 \cdot p q)] \\
= & \frac{1}{2}\left[p^{2} q^{2}+p q-2 \cdot p-2 \cdot q+2\right] \\
= & {\left[\frac{p^{2} q^{2}-p q}{2}+p q-p-q+1\right] } \\
= & {\left[\left(\begin{array}{c}
p q \\
2
\end{array}\right)-\phi(p q)\right]+2 \cdot \phi(p q) }
\end{aligned}
$$

On the other hand;

$$
W\left(P\left(Z_{p q}\right)\right)=p q(p q-1)-m=\left(\begin{array}{c}
p q \\
2
\end{array}\right)+\phi(p q)
$$

where

$$
m=\left(\begin{array}{c}
p q \\
2
\end{array}\right)-\phi(p q)
$$

$\left({ }^{* *}\right)$ equation put in $\left(^{*}\right)$ equation, we obtain,

$$
W\left(P\left(Z_{p q}\right)\right)=m+2 \phi(p q) .
$$

This completes the proof. 
On the other hand using $m$ in $\left.{ }^{* *}\right)$, we obtain

$$
\begin{aligned}
W\left(P\left(Z_{p q}\right)\right) & =m+2 \phi(p q) \\
& =\left(\begin{array}{c}
p q \\
2
\end{array}\right)-\phi(p q)+2 \phi(p q) \\
& =\left(\begin{array}{c}
p q \\
2
\end{array}\right)+\phi(p q)
\end{aligned}
$$

This completes the proof.

Theorem 11. If $P\left(Z_{n}\right)$ is a power graph of order $n=p^{k}$ or $n=p q$ and $m$ edges, where $p$ and $q$ are distinct prime and $k$ is a nonnegative integer. Then

$$
\operatorname{maks}\left\{W\left(P\left(Z_{n}\right)\right)\right\}=\left(\begin{array}{c}
n+1 \\
2
\end{array}\right)
$$

and

$$
\min \left\{W\left(P\left(Z_{n}\right)\right)\right\}=\left(\begin{array}{l}
n \\
2
\end{array}\right)
$$

Proof. If $n=p^{k}$ in Corollary 6 .

$$
W\left(P\left(Z_{p^{k}}\right)\right)=\left(\begin{array}{c}
p^{k} \\
2
\end{array}\right) .
$$

And so

$$
\min \left\{W\left(P\left(Z_{n}\right)\right)\right\}=\left(\begin{array}{l}
n \\
2
\end{array}\right)
$$

And if $n=p q$ in Corollary 10.

$$
W\left(P\left(Z_{p q}\right)\right)=\left(\begin{array}{c}
p q \\
2
\end{array}\right)+\phi(p q)
$$

therefore

$$
W\left(P\left(Z_{n}\right)\right) \leq\left(\begin{array}{l}
n \\
2
\end{array}\right)+\phi(n) .
$$

Also

$$
\phi(n) \leq n
$$

We write

$$
W\left(P\left(Z_{n}\right)\right) \leq\left(\begin{array}{l}
n \\
2
\end{array}\right)+\phi(n) \leq\left(\begin{array}{l}
n \\
2
\end{array}\right)+n .
$$

And so,

$$
\operatorname{maks}\left\{W\left(P\left(Z_{n}\right)\right)\right\}=\left(\begin{array}{c}
n+1 \\
2
\end{array}\right) .
$$

Theorem 12. If $P\left(Z_{n}\right)$ is a power graph of order $n=p^{k}$ and $m$ edges, where $p$ is prime and $k$ is a nonnegative integer. Then

$$
W_{e}\left(P\left(Z_{n}\right)\right)=3\left\{\left(\begin{array}{l}
n \\
3
\end{array}\right)+\operatorname{diam}\left(L\left(P\left(Z_{n}\right)\right)\right)\left(\begin{array}{l}
n \\
4
\end{array}\right)\right\} \text {. }
$$


Proof. For $P\left(Z_{p^{k}}\right)$ power graph, $E\left(P\left(Z_{n}\right)\right)=\left(\begin{array}{l}n \\ 2\end{array}\right)$ and $\forall u \in V\left(P\left(Z_{n}\right)\right)$, $d(u)=n-1$.

Let's consider to this figure in $P\left(Z_{p^{k}}\right)$ power graph any $e_{\bar{n}, \overline{n-1}} \in E\left(P\left(Z_{p^{k}}\right)\right)$. For $P\left(Z_{p^{k}}\right)$ power graph of Line graph as shown in Figure 1 .

Choose the random $e_{\bar{n}, \overline{n-1}} \in E\left(P\left(Z_{p^{k}}\right)\right)$ edge and this corner in neighborhood $L\left(P\left(Z_{n}\right)\right)$ line graph in Figure 2. In the same way, with $e_{\bar{n}, \overline{n-1}} \in V\left(L\left(P\left(Z_{p^{k}}\right)\right)\right)$ point neighborhood amount of points $2(n-2)$. In the same way $e_{\bar{n}, \overline{n-1}}$ neighborhood with corner amount of point $m-1-2(n-2)$ and therefore $V\left(L\left(P\left(Z_{p^{k}}\right)\right)\right)$ if each elements for calculated and if edge-Wiener index identified we have the following result.

In edge-Wiener index

$$
\begin{aligned}
W_{e}\left(P\left(Z_{p^{k}}\right)\right)=\frac{1}{2} \sum_{\{e, f\} \subseteq E\left(P\left(Z_{n}\right)\right)} d(e, f) \\
=\frac{1}{2}\left\{\sum_{u v=e}[(d(u)+d(v)-2)]\right. \\
\left.+\sum_{u v=e}\left[\operatorname{diam}\left(L\left(P\left(Z_{n}\right)\right)\right) \cdot((m-1)-(d(u)+d(v)-2))\right]\right\} \\
=\frac{1}{2}\left\{\left(\begin{array}{l}
n \\
2
\end{array}\right)\left[2(n-2)+\operatorname{diam}\left(L\left(P\left(Z_{n}\right)\right)\right)\left(\left(\begin{array}{l}
n \\
2
\end{array}\right)-1-2(n-2)\right)\right]\right\} \\
=\left[\frac{n(n-1)(n-2)}{2}+\frac{n(n-1)}{4} \operatorname{diam}\left(L\left(P\left(Z_{n}\right)\right)\right)\left(\frac{n^{2}-5 n-6}{2}\right)\right] \\
=3\left(\begin{array}{l}
n \\
3
\end{array}\right)+\frac{n(n-1)(n-2)(n-3)}{8} \operatorname{diam}\left(L\left(P\left(Z_{n}\right)\right)\right) \\
W_{e}\left(P\left(Z_{p^{k}}\right)\right)=3\left[\left(\begin{array}{l}
n \\
3
\end{array}\right)+\operatorname{diam}\left(L\left(P\left(Z_{n}\right)\right)\right)\left(\begin{array}{l}
n \\
4
\end{array}\right)\right]
\end{aligned}
$$

Concluded, namely the prove end.

Theorem 13. If $P\left(Z_{n}\right)$ is a power graph of order $n=p^{k}$ and $m$ edges, where $p$ is prime and $k$ is a nonnegative integer. Then

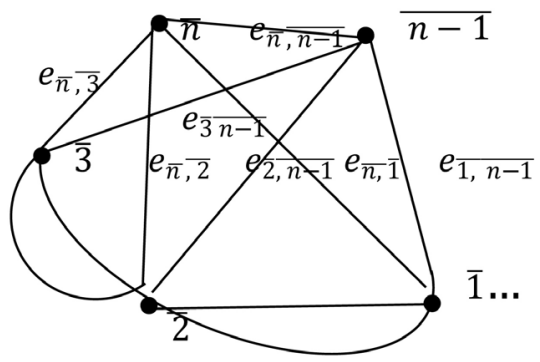

Figure 1. Power grap of $Z_{p^{k}}$. 


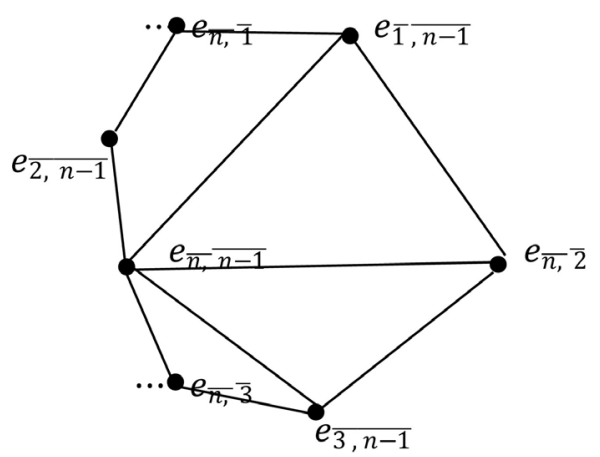

Figure 2. Line graph of $P\left(Z_{n}\right)$.

$$
W_{e}\left(P\left(Z_{n}\right)\right)=\left(\begin{array}{c}
n-1 \\
2
\end{array}\right) W\left(P\left(Z_{n}\right)\right)
$$

Proof. $n=p^{k} \quad\left(\in Z^{+}\right)$is in $W\left(P\left(Z_{n}\right)\right)=\left(\begin{array}{l}n \\ 2\end{array}\right)$. In the same way,

Case 1. for $n=2,3$ and according to $\operatorname{diam}\left(L\left(P\left(Z_{n}\right)\right)\right)=1, W_{e}\left(P\left(Z_{2}\right)\right)=0$, therefore $W_{e}\left(P\left(Z_{3}\right)\right)=W\left(P\left(Z_{3}\right)\right)$ ve $\left(\begin{array}{c}3-1 \\ 2\end{array}\right)=1$, namely this equation the proof.

Case 2. For $n \neq 2,3$ is $\operatorname{diam}\left(L\left(P\left(Z_{n}\right)\right)\right)=2$ in theorem 12.,

$$
\begin{aligned}
W_{e}\left(P\left(Z_{n}\right)\right) & =3\left[\left(\begin{array}{l}
n \\
3
\end{array}\right)+\operatorname{diam}\left(L\left(P\left(Z_{n}\right)\right)\right)\left(\begin{array}{l}
n \\
4
\end{array}\right)\right] \\
& =3\left[\left(\begin{array}{l}
n \\
3
\end{array}\right)+2\left(\begin{array}{l}
n \\
4
\end{array}\right)\right] \\
& =\frac{1}{2} n(n-1)\left[(n-2)+\left(\frac{(n-2)(n-3)}{2}\right)\right] \\
& =\left(\begin{array}{c}
n \\
2
\end{array}\right)(n-2)\left[1+\frac{n-3}{2}\right] \\
& =\left(\begin{array}{c}
n-1 \\
2
\end{array}\right) W\left(P\left(Z_{n}\right)\right)
\end{aligned}
$$

Thus the proof is completed.

\section{Conclusion}

We will show the undirected power graph of a Group $G$ with $P(G)$. Here, the undirected $P\left(Z_{n}\right)$ Power graph of the group $\left(Z_{n},+\right)$ according to $N=p^{k}$ and $n=$ $p q$, with $p, q$ being different primes and $k$ being positive integers, is considered and new theorems and results on the Wiener index calculations of these power graphs with the help of Euler function are have been obtained.

\section{Acknowledgements}

This paper is derived from the first author's PH's thesis. 


\section{Conflicts of Interest}

The authors declare no conflicts of interest regarding the publication of this paper.

\section{References}

[1] Alhevaz, A., Baghipuri, M. and Shang, Y. (2019) On Generalized Distance Gaussian Estrada Index of Graphs. Symmetry, 11, 1276. https://doi.org/10.3390/sym11101276

[2] Iran Manesh, A., Gutman, I., Khormali, O. and Mahmiani, A. (2009) The Edge Versions of the Wiener Index. MATCH Communications in Mathematical and in Computer Chemistry, 61, 663-672.

[3] Chattopadhyay, S. and Panigrahi, P. (2017) On Sum of Powers of the Laplacian Eigenvalues of Power Graphs of Certain Finite Groups. Electronic Notes in Discrete Mathematics, 63, 137-143.

[4] Walikar, H.B., Shigehalli, V.S. and Ramane, H.S. (2004) Bounds on the Wiener Index of a Graph. MATCH Communications in Mathematical and in Computer Chemistry, 50, 117-132.

[5] Wiener, H. (1947) Structural Determination of Paraffin Boiling Points, Journal of the American Chemical Society, 69, 17-20.

[6] Chakrabarty, I. Ghosh, S. and Sen, M.K. (2009) Undirected Power Graphs of Semigroups. Semigroup Forum, 78, 410-426. 